\title{
Survival and cessation in injecting drug users: prospective observational study of outcomes and effect of opiate substitution treatment
}

\author{
Jo Kimber, NHMRC postdoctoral fellow, ${ }^{1,2}$ Lorraine Copeland, researcher, ${ }^{3}$ Matthew Hickman, professor in \\ public health and epidemiology, ${ }^{1}$ John Macleod, professor in clinical epidemiology and primary care, ${ }^{2}$ James \\ McKenzie, research community psychiatry nurse, ${ }^{3}$ Daniela De Angelis, senior statistician, ${ }^{4,5}$ James Roy \\ Robertson, reader and general practice principal ${ }^{3,6}$
}

Department of Social Medicine, University of Bristol, Bristol BS8 2PS

${ }^{2}$ National Centre in HIV Epidemiology and Clinical Research, University of New South Wales, Sydney 2052, Australia

${ }^{3}$ Muirhouse Medical Group, Edinburgh EH4 4P

${ }^{4}$ MRC Biostatistics Unit, Institute of Public Health, University of Cambridge

${ }^{5}$ Health Protection Agency, Centre for Infections, London

${ }^{6}$ Division of Community Health Sciences, University of Edinburgh, Edinburgh EH8 9AG

Correspondence to: $\mathrm{M}$ Hickman matthew.hickman@bristol.ac.uk

Cite this as: $B M J$ 2010;340:c3172 doi:10.1136/bmj.c3172

\section{ABSTRACT}

Objectives To examine survival and long term cessation of injecting in a cohort of drug users and to assess the influence of opiate substitution treatment on these outcomes.

Design Prospective open cohort study.

Setting A single primary care facility in Edinburgh.

Participants 794 patients with a history of injecting drug use presenting between 1980 and 2007; 655 (82\%) were followed up by interview or linkage to primary care records and mortality register, or both, and contributed 10390 person years at risk; 557 (85\%) had received opiate substitution treatment.

Main outcome measures Duration of injecting: years from first injection to long term cessation, defined as last injection before period of five years of non-injecting; mortality before cessation; overall survival.

Results In the entire cohort 277 participants achieved long term cessation of injecting, and 228 died. Half of the survivors had poor health related quality of life. Median duration from first injection to death was 24 years for participants with HIV and 41 years for those without HIV. For each additional year of opiate substitution treatment the hazard of death before long term cessation fell $13 \%$ (95\% confidence interval $17 \%$ to $9 \%$ ) after adjustment for HIV, sex, calendar period, age at first injection, and history of prison and overdose. Conversely exposure to opiate substitution treatment was inversely related to the chances of achieving long term cessation.

Conclusions Opiate substitution treatment in injecting drug users in primary care reduces this risk of mortality, with survival benefits increasing with cumulative exposure to treatment. Treatment does not reduce the overall duration of injecting.

\section{INTRODUCTION}

Injection drug use is an important public health problem with a prevalence of around 1-2\% among young adults in the United Kingdom and a standardised mortality ratio over 10 times that of the general population. ${ }^{1}$ Deaths in those who inject opiates are mainly a consequence of overdose and bloodborne infection. ${ }^{2}$ The principal treatment for dependent users is opiate substitution therapy, commonly oral methadone, ${ }^{3}$ which in the UK is mostly delivered in primary care settings. Opiate substitution treatment can reduce opiate use, mortality, and transmission of bloodborne infections, though most evidence comes from relatively short term studies. ${ }^{4-8}$

Short periods of cessation from injecting are relatively common, ${ }^{9}$ but few studies have long enough follow-up to observe long term cessation, and the impact of opiate substitution treatment on the overall duration of injecting is unclear. ${ }^{10}$

We report on a follow-up study of the Edinburgh addiction cohort. ${ }^{11}$ This study included injecting drug users, most of whom were using heroin, recruited through Muirhouse Medical Group, a single primary care facility in a deprived area of Edinburgh, during a rapid local HIV epidemic. ${ }^{12}$ We describe the duration of injecting and survival and assess the influence of opiate substitution treatment and other factors on these outcomes.

\section{METHODS}

\section{Data source}

Methods are described in detail elsewhere. ${ }^{1113}$ Briefly, between 1980 and 2006 all patients at a large primary care facility in Edinburgh who reported a history of injecting drug use were recruited to the study. Opiate substitution treatment was publicly funded and accessible to patients throughout the study period, in keeping with national guidelines. Cohort members were flagged with the General Register Office for Scotland to allow for tracing of deaths and changes of general practitioner. From October 2005 to November 2007 we attempted to contact all surviving cohort members to conduct a follow-up interview. Information was also collected from primary care notes when these were available. 
Table 1|Characteristics at recruitment and follow-up in 794 participants in Edinburgh addiction cohort. Figures are numbers (percentages), unless stated otherwise

\begin{tabular}{lc} 
Characteristic & Data \\
Male & $543(68)$ \\
\hline Calendar period of recruitment: & $361(46)$ \\
\hline $1980-9$ & $201(25)$ \\
\hline $1990-9$ & $231(29)$ \\
\hline $2000-7$ & $19.9(5.1)$ \\
\hline Mean (SD) age at first injection (years) & $26.7(6.3)$ \\
\hline Mean (SD) age at recruitment (years) & $10.2(6.8,<1-25)$ \\
\hline Mean (SD, range) years of follow-up & $132(54)$ \\
\hline Lnterviewed at follow-up & $228(29)$ \\
\hline Dead to follow-up &
\end{tabular}

The cohort comprised 794 participants, of whom 571 were still alive at the start of follow-up and 432 $(75 \%)$ were interviewed. By the end of follow-up, five of those we interviewed had died, bringing the total number of deaths to 228 (29\%). We were able to follow up $655(82 \%)$ using primary care records, including for $187(82 \%)$ of those who died. One hundred and thirty nine $(18 \%)$ participants were lost to follow-up. ${ }^{11}$

\section{Instruments}

We used a coding sheet to systematically extract data on clinical history from primary care records. The causes of death were transcribed from the death certificates provided by national mortality registers and grouped into the underlying cause of death from HIV, external injury, liver disease, and other causes. We developed a life grid $^{14}$ questionnaire administered by an interviewer to collect retrospective self reported data on injecting drug use over a lifetime ${ }^{11}$ and assessed current health status based on the alcohol use disorders identification test (AUDIT), ${ }^{15}$ the hospital anxiety and depression scale (HADS), ${ }^{16}$ and a self rated visual analogue scale of current health state (EQ-VAS). ${ }^{17}$

\section{Primary outcomes}

Time to event variables were duration in years from first injection to death (from primary care records) or the start of a period of long term cessation of injecting (from follow-up interviews). ${ }^{11}$ We defined long term cessation as at least five consecutive years without injecting before the last follow-up or death as this was consistent with other cohorts where the probability of relapse was relatively low after five years. ${ }^{18-20}$ In our study, for the 377 interviewees with a history of cessation of injecting, 111 relapsed after their last cessation of at least three months, and $97(87 \%)$ did so within five years (range $0.25-23.25$ years).

\section{Exposure variables}

The main exposure variable was receipt of opiate substitution treatment (such as oral methadone, buprenorphine, dihydrocodeine $)^{21}$ ascertained from primary care records. Data were available on cumulative months and years of exposure from year of injection to the outcome (long term cessation or death). Opiate substitution treatment in the period defining long term cessation (five years after last injection) was included in the exposure variable, with treatment after this period excluded.

As dead participants were included in both analyses, in addition to opiate substitution treatment we considered the following covariates extracted from primary care notes: sex, age at first injection, calendar year at first injection (<1986, 1986), HIV status (negative, positive), periods in prison (none, once, more than once); and clinical history (no, yes) of overdose requiring treatment at an accident and emergency department, problem drinking, referrals for serious mental health issues, and self harm.

\section{Analysis procedures}

We used discrete time survival models for the analysis of the time from starting injecting to death or long term cessation because the information on the times of onset and cessation events was available only in whole years. ${ }^{2223}$ Firstly, overall survival was analysed with a logistic model. Secondly, time to death before long term cessation and time to long term cessation were analysed with a competing risk model. ${ }^{24}$ The competing risk approach was required because the occurrence of the death event prevents an individual from experiencing long term cessation and vice versa. Moreover, this framework enabled the assessment of the potentially different role of covariates on the probability of occurrence of the two competing events, allowing a better understanding of each cause of outcome. We used a multinomial logistic hazard model to model the log odds of death before long term cessation and long term cessation simultaneously. ${ }^{2526}$ Individuals contribute one year of exposure from when they start injecting to the time they experience one of the events or are censored at interview.

We used dummy variables representing years from first injection to estimate (non-parametrically) time varying hazards, in both the logistic and multinomial logistic models. We used a complete case analysis approach as we had insufficient information on nonresponders to impute missing data. We excluded the 41 surviving participants with less than six years' follow-up since their first injection from the competing risk analysis because they had insufficient follow-up to measure long term cessation. The best fitting multinomial logistic model included duration variables of 1 year, 2-10 years, 11-15 years, and 16-38 years from initiation of injecting and the opiate substitution treatment and age at onset of injecting variables as continuous variables. We fitted and found evidence for an interaction between opiate substitution treatment and duration, suggesting that the effect of treatment differed over time (likelihood ratio test $\chi^{2}=36.19, \mathrm{df}=6$, $\mathrm{P}<0.0001)$. As none of the individual interactions between opiate substitution treatment and duration were significant for survival $(r=-0.15$ (SE 0.27), $\mathrm{P}=0.57$, for $2-10$ years' duration; $r=-0.07$ (SE 0.26), 
Table 2 | Injecting and health status of interviewees in Edinburgh addiction cohort at followup. Figures are numbers (percentages)

\begin{tabular}{|c|c|c|c|c|}
\hline \multirow[b]{2}{*}{ Characteristic } & \multirow[b]{2}{*}{$\begin{array}{c}\text { Total } \\
(n=432)\end{array}$} & \multicolumn{3}{|c|}{ Long term cessation of injecting* } \\
\hline & & $\begin{array}{l}\text { Not achieved } \\
\quad(n=241)\end{array}$ & $\begin{array}{l}\text { Achieved } \\
(n=165)\end{array}$ & $\begin{array}{l}\text { P value for difference } \\
\qquad\left(x^{2} \text { test }\right)\end{array}$ \\
\hline Current injecting drug users & $135(31)$ & $135(56)$ & - & - \\
\hline $\begin{array}{l}\text { Current opiate substitution } \\
\text { treatment }\end{array}$ & $302(70)$ & $196(81)$ & $106(64)$ & $<0.05$ \\
\hline Smoker & 403 (93) & $229(95)$ & $149(90)$ & $>0.05$ \\
\hline Problem drinker† & $87(20)$ & $55(23)$ & 25(15) & $>0.05$ \\
\hline Anxious & $209(48)$ & $128(53)$ & $71(43)$ & $<0.05$ \\
\hline Depressed§ & $114(26)$ & $70(29)$ & $40(24)$ & $>0.05$ \\
\hline Low subjective QoLT & $226(52)$ & $144(60)$ & $82(50)$ & $>0.05$ \\
\hline
\end{tabular}

*Sample restricted to 406 participants with $>5$ years' observation since their first injection.

†AUDIT score of $\geq 16$, indicating high risk or harmful drinking in past year. ${ }^{15}$

fHADS anxiety subscale score $\geq 11$, indicating caseness. ${ }^{16}$

\$HADS depression subscale score $\geq 11$, indicating caseness. ${ }^{16}$

IEqVAS $z$ score $<-1.96$ based on UK population norms by age, sex, and smoking status. ${ }^{40}$

$\mathrm{P}=0.78$, for $11-15$ years' duration; $r=0.05$ (SE 0.26), $\mathrm{P}=0.18$, for $16-38$ years' duration), we limited the interaction to long term cessation only. All analyses were conducted with Stata statistical software (version 10.1)

\section{RESULTS}

Table 1 shows characteristics of the cohort at recruitment. Table 2 shows follow-up outcomes and current drug use and health status of interviewees.

Among interviewees, $302(70 \%)$ were currently receiving opiate substitution treatment, primarily methadone; 135 (31\%) were currently injecting, of whom $112(83 \%)$ were also receiving opiate substitution treatment; and $165(38 \%)$ had achieved long term cessation, of whom 106 (64\%) were currently receiving opiate substitution treatment. Most interviewees were current smokers (403, 93\%). For other measures, 20\% (87) were problem drinkers, 48\% (209) were anxious, $26 \%$ (114) were depressed, and 52\% (226) had poor health related quality of life. Interviewees who achieved long term cessation had lower levels of morbidity.

Among the 654 with data extracted from primary care case notes, $558(85 \%)$ had a history of opiate substitution treatment: $79 \%(439)$ had received methadone, 73\% (410) dihydrocodeine, 7\% (37) buprenorphine, and 1\% (8) other opiates; 314 patients had had multiple forms of opiate substitution treatment. A history of periods in prison was recorded in primary care notes for $360(55 \%) ; 281(43 \%)$ had a history of problem drinking and $237(36 \%)$ had a history of overdose requiring hospital treatment; 199 (30\%) had been referred to specialist mental health services; and $196(30 \%)$ had a history of self harm. In total, 189 (29\%) participants were HIV antibody positive.

By the study end point, $228(29 \%)$ participants had died (table 3). The leading causes of death were HIV $(102,45 \%)$, drug overdose $(55,24 \%)$, and liver disease/ injury $(37,16 \%)$. More than three quarters of deaths from drug overdose were among those who did not achieve long term cessation (78\%), and drug overdose deaths accounted for $38 \%$ (43) of all deaths in that group. Most deaths could be attributed to injecting drug use (table 3), with many of the remaining deaths due to associated problems with other substance use, particularly tobacco and alcohol, or comorbid mental illness.

Overall survival and competing risks: survival and long term cessation

Figure 1 shows survival by HIV status. Median survival for HIV positive patients was 24 years compared with 41 years for HIV negative patients (for patients set to median exposure to opiate substitution treatment, median age at first injection, onset $<1986$, no prison history, and no history of overdose).

Among those who died and interviewees whose first injection had been at least six years before follow-up ( $\mathrm{n}=566), 49 \%$ (277) achieved long term cessation, 16\% (91) died before achieving long term cessation, and 35\% (198) survived but had not achieved long term cessation by follow-up. Of those who died before achieving long term cessation, within 25 years after their first injection, half of HIV positive participants had died compared with 10\% (9) of HIV negative participants. Opiate substitution treatment was associated with increased survival (that is, decreased time to death). Table 4 shows that for each year of opiate substitution treatment, the probability of death was reduced by $10 \%$. Without adjustment for other factors, survival was also reduced in those with a prison history and history of overdose and was negatively associated with age at onset of injecting (table 4). There was no difference in survival by sex or history of alcohol problems, self harm, or serious mental illness. Evidence for improved survival with opiate substitution treatment remained after adjustment (for HIV, history of

Table 3 | Primary cause of death in Edinburgh addiction cohort. Figures are numbers (percentages) of participants

\begin{tabular}{lcc} 
HIV & $\begin{array}{c}\text { All deaths } \\
(\mathrm{n}=228) \\
\text { Injury: }\end{array}$ & $\begin{array}{c}\text { Deaths before long term } \\
\text { cessation }(\mathrm{n}=112)\end{array}$ \\
\hline Drug overdose* & $43(38)$ \\
\hline Suicide† & $55(24)$ & $43(38)$ \\
\hline Homicide & $15(7)$ & $8(7)$ \\
\hline Liver: & $1((1)$ & 0 \\
\hline Liver disease & & $8(7)$ \\
\hline Alcohol related & $26(11)$ & $2(2)$ \\
\hline Other causes: & $11(5)$ & $4(4)$ \\
\hline Cardiovascular disease & $7(3)$ & $2(2)$ \\
\hline Injecting related & $2(1)$ & $1(1)$ \\
\hline Lung/throat cancer & $2(1)$ & 0 \\
\hline Respiratory disease & $2(1)$ & $1(1)$ \\
\hline Unascertained & $2(1)$ & 0 \\
\hline Other & $3(1)$ & \\
\hline
\end{tabular}

*Including 12 deaths from ingested substances after long term injecting cessation.

†Six of 15 suicides were drug overdoses classified as intentional self harm: paracetamol $(n=3)$, insulin $(n=1)$, nifedipine $(n=1)$, dihydrocodeine and alcohol $(n=1)$. 


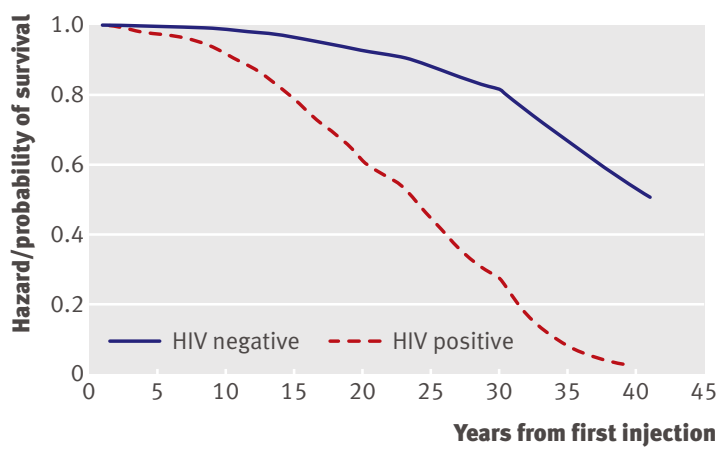

Fig 1| Overall survival from year of first injection to death by HIV status

overdose, prison history, age at first injection, calendar period of onset), with the hazard of death reduced by $13 \%$ (95\% confidence interval $17 \%$ to $9 \%, \mathrm{P}<0.001)$. Figure 2 shows that for patients who do not start opiate substitution treatment (unexposed), a quarter will be dead within 25 years of their first injection compared with $6 \%$ of those with more than five cumulative years of exposure to opiate substitution treatment (with other factors set to HIV negative, median age at first injection, onset $<1986$, no prison history, no history of overdose). After adjustment for opiate substitution treatment and other covariates (see above), the probability of death before long term cessation was increased almost sixfold (3.5 to 10.1) for those infected with HIV and twofold (1.3 to 3.1) for those with a history of heroin overdose and the effect of prison history on survival was diluted. The effect of calendar period (onset of injecting from 1986) on survival switched from insufficient or weak evidence of a protective effect (hazard ratio $0.74, \mathrm{P}=0.22$ ) to evidence of a more than twofold increase in the probability of death before long term cessation ( 1.1 to $4.0, \mathrm{P}=0.02$ ) after adjustment for HIV infection in particular and other covariates (opiate substitution treatment, history of overdose, age at first injection, prison history, sex).

Opiate substitution treatment was associated with an increased duration of injecting (that is, time to long term cessation): for each year of treatment, before adjustment, duration was increased by $11 \%$ (table 4 ). Table 4 shows also that after adjustment for key covariates (sex, HIV infection, age at first injection, calendar period of onset, prison history, history of overdose) and an interaction between opiate substitution treatment and time, the impact of opiate substitution treatment on duration wanes over time - that is, decreasing the probability of cessation by 0.73 ( 0.65 to 0.81$)$ in the first year and then, as the interactions are all greater than 1 , by 0.89 in years $2-10,0.95$ in years $11-15$, and 0.91 in years $16-38$. (The latter are generated by multiplying the interaction terms with the effect of opiate substitution treatment.) Figure 3 shows that for patients who did not start opiate substitution treatment, the median duration of injecting was five years (with nearly $30 \%$ ceasing within a year) compared with 20 years for those with more than five years of exposure to treatment (with other factors set to HIV negative, median age at first injection, onset $<1986$, no prison history, no history of overdose). Figure 3 also shows that the difference in the probability of long term cessation between those who do and those who do not receive opiate substitution treatment narrows over time.

Table 4 also shows that the probability of cessation, after adjustment for opiate substitution treatment and other covariates in the model, was lower for injecting drug users with more than one period of prison recorded $(0.57,0.42$ to 0.78$)$ and for those injecting from $1986(0.54,0.38$ to 0.77$)$. There was weak evidence that women had a $30 \%$ higher probability of achieving long term cessation, but after adjustment the difference was diluted $(1.11,0.84$ to 1.47$)$. Age at onset was associated with a $6 \%(3 \%$ to $9 \%)$ higher probability of cessation; and there was weak evidence that HIV infection was also associated with a higher probability of cessation (1.32, 0.99 to 1.75$)$.

Based on data available from the 369 interviewed patients, those exposed to opiate substitution treatment reported injecting less frequently while receiving treatment compared with periods out of treatment (mean 157 v 273 days a year, $t=-3.9$, df=171, $\mathrm{P}<0.001)$. For those who achieved long term cessation, however, the number of total days injecting tended to be lower among those who were not exposed to opiate substitution treatment compared with those who were exposed (878 $v 1469$ days, $t=-0.9, \mathrm{df}=140, \mathrm{P}=0.36$ ).

\section{DISCUSSION}

This follow-up study of the Edinburgh addiction cohort shows the chronic nature and multiple adverse health consequences of injecting drug use. ${ }^{27}$ In our cohort of injectors recruited from primary care around half of those infected with HIV will die within 24 years of starting injecting, and even for those without HIV median survival was 41 years, suggesting half of those who start injecting in late adolescence will be dead by middle age. Quality of life and health status of surviving injecting drug users also was poor.

Our results confirm the beneficial effects of opiate substitution treatment delivered in routine primary care over long periods. We found a dose-response relation between exposure to such treatment and survival before long term cessation. The overall median duration of injecting, however, was longer for injecting drug users who were exposed to opiate substitution treatment. It is argued that this treatment confers its health benefit through promoting injection cessation. ${ }^{328}$ Our data did not support this hypothesis and suggest that it conferred health benefits irrespective of whether injecting drug users continued injecting, though users injected less often when receiving treatment, as consistently shown in clinical trials and observational studies. ${ }^{29}$ Nonetheless, the cumulative total number of injections is probably greater in those exposed to opiate substitution treatment (as a high proportion of those who did not receive such treatment stop injecting within the first year of onset). Opiate substitution 
Table 4 Competing risk (hazard) from year of first injection to achieving long term cessation or death before long term cessation in participants in Edinburgh addiction cohort whose first injection had been at least six years before follow-up (multinomial logistic regression model)

\begin{tabular}{|c|c|c|c|c|c|c|c|c|c|c|}
\hline \multirow{3}{*}{$\begin{array}{l}\text { Variable (No in } \\
\text { group) }\end{array}$} & \multicolumn{5}{|c|}{ Died before achieving long term cessation } & \multicolumn{5}{|c|}{ Long term cessation achieved before death/follow up } \\
\hline & \multirow[b]{2}{*}{ No (\%) } & \multicolumn{4}{|c|}{ Hazard ratio $(95 \% \mathrm{Cl})$} & \multirow[b]{2}{*}{ No (\%) } & \multicolumn{4}{|c|}{ Hazard ratio $(95 \% \mathrm{Cl})$} \\
\hline & & Unadjusted & $P$ value & Adjusted* & $P$ value & & Unadjusted & $P$ value & Adjusted* & $P$ value \\
\hline \multicolumn{11}{|l|}{ Sex: } \\
\hline Male (387) & $67(17)$ & 1.00 & - & - & - & $184(48)$ & 1.00 & - & - & - \\
\hline Female (179) & $25(14)$ & $0.96(0.60$ to 1.52$)$ & 0.86 & 1.09 (0.67 to 1.79$)$ & 0.73 & $94(52)$ & 1.30 (1.01 to 1.68$)$ & 0.05 & 1.11 (0.84 to 1.47$)$ & 0.45 \\
\hline \multicolumn{11}{|c|}{ Age first injection (median=18, IQR=16-22): } \\
\hline Age & - & 1.00 & - & - & - & - & 1.00 & - & - & - \\
\hline 1 year later & - & 1.05 (1.01 to 1.10$)$ & 0.03 & 1.09 (1.04 to 1.15$)$ & $<0.001$ & - & 1.06 (1.04 to 1.09$)$ & 0.00 & 1.06 (1.03 to 1.09$)$ & 0.00 \\
\hline \multicolumn{11}{|c|}{ Year of first injection: } \\
\hline <1986 (351) & $69(20)$ & 1.00 & - & - & - & $220(63)$ & 1.00 & - & - & - \\
\hline$\geq 1986$ (215) & $23(11)$ & $0.74(0.46$ to 1.20$)$ & 0.22 & $2.13(1.13$ to 4.01$)$ & 0.02 & $57(27)$ & $0.58(0.43$ to 0.78$)$ & 0.00 & 0.54 (0.38 to 0.77$)$ & 0.00 \\
\hline \multicolumn{11}{|c|}{ Years of exposure to opiate substitution treatment (median=4.3, IQR=0-8.5): } \\
\hline None & - & 1.00 & - & - & - & - & 1.00 & - & - & - \\
\hline $\begin{array}{l}\text { For each year of } \\
\text { exposure }\end{array}$ & - & $0.90(0.87$ to 0.94$)$ & $<0.001$ & 0.87 (0.83 to 0.91$)$ & $<0.001$ & - & $0.89(0.86$ to 0.91$)$ & $<0.001$ & $0.73(0.65$ to 0.81$)$ & $<0.001$ \\
\hline \multicolumn{11}{|l|}{ Prison exposure: } \\
\hline None (267) & $27(10)$ & 1.00 & - & - & - & $152(57)$ & 1.00 & - & - & - \\
\hline$\leq 1$ year (109) & $17(16)$ & 1.31 (0.71 to 2.42$)$ & 0.38 & $1.31(0.70$ to 2.46$)$ & 0.39 & $48(44)$ & $0.66(0.47$ to 0.92$)$ & 0.01 & $0.81(0.57$ to 1.14$)$ & 0.23 \\
\hline$>1$ year (190) & $48(25)$ & 1.98 (1.23 to 3.19$)$ & 0.01 & $1.39(0.84$ to 2.32$)$ & 0.20 & $77(41)$ & $0.56(0.43$ to 0.75$)$ & $<0.001$ & $0.57(0.42$ to 0.78$)$ & $<0.001$ \\
\hline \multicolumn{11}{|c|}{ Clinical history of overdose: } \\
\hline No (352) & $43(12)$ & 1.00 & - & - & - & $193(55)$ & 1.00 & - & - & - \\
\hline Yes (214) & $49(23)$ & 1.68 (1.11 to 2.54$)$ & 0.01 & 2.00 (1.29 to 3.12$)$ & $<0.001$ & $84(39)$ & 0.64 (0.49 to 0.83$)$ & $<0.001$ & 0.82 (0.63 to 1.09 ) & 0.17 \\
\hline \multicolumn{11}{|l|}{ HIV status: } \\
\hline No (384) & $35(9)$ & 1.00 & - & - & - & $162(42)$ & 1.00 & - & - & - \\
\hline Yes(182) & $57(31)$ & 3.81 (2.49 to 5.83$)$ & $<0.001$ & $\begin{array}{c}5.97 \text { (3.53 to } \\
10.12)\end{array}$ & $<0.001$ & $115(63)$ & 1.66 (1.30 to 2.12$)$ & $<0.001$ & 1.32 (0.99 to 1.75$)$ & 0.06 \\
\hline
\end{tabular}

Clinical history of alcohol problems:

\begin{tabular}{|c|c|c|c|c|c|c|c|c|c|c|}
\hline No (315) & $43(14)$ & 1.00 & - & - & - & $158(50)$ & 1.00 & - & - & - \\
\hline Yes (251) & $49(20)$ & 1.23 (0.81 to 1.86$)$ & 0.33 & - & - & $119(47)$ & 0.81 (0.64 to 1.04$)$ & 0.10 & - & - \\
\hline \multicolumn{11}{|c|}{ Clinical history of serious mental health: } \\
\hline No (389) & $64(16)$ & 1.00 & - & - & - & $180(46)$ & 1.00 & - & - & - \\
\hline Yes (177) & $28(16)$ & $1.00(0.64$ to 1.57$)$ & 0.99 & - & - & $97(55)$ & 1.23 (0.96 to 1.59$)$ & 0.10 & - & - \\
\hline \multicolumn{11}{|c|}{ Clinical history of self harm: } \\
\hline No (392) & $63(16)$ & 1.00 & - & - & - & $197(50)$ & 1.00 & - & - & - \\
\hline Yes (174) & $29(17)$ & $1.12(0.72$ to 1.75$)$ & 0.61 & - & - & $80(46)$ & 0.99 (0.76 to 1.29$)$ & 0.94 & - & - \\
\hline \multicolumn{11}{|c|}{ Years to event (from first injection to death or long term cessation): } \\
\hline$<1-1(67)$ & $1(1)$ & - & - & $\begin{array}{c}0.0001(0.00001 \\
\text { to } 0.001)\end{array}$ & $<0.001$ & $66(99)$ & - & - & $0.1(0.07$ to 0.3$)$ & $<0.001$ \\
\hline $2-10(222)$ & $33(15)$ & - & - & $\begin{array}{c}0.0005(0.0002 \text { to } \\
0.002)\end{array}$ & $<0.001$ & $122(55)$ & - & - & 0.03 (0.01 to 0.05$)$ & $<0.001$ \\
\hline $11-15(115)$ & $24(21)$ & - & - & $\begin{array}{c}0.002(0.0006 \text { to } \\
0.007)\end{array}$ & $<0.001$ & $51(44)$ & - & - & 0.03 (0.02 to 0.07$)$ & $<0.001$ \\
\hline $16-38(110)$ & 34 (31) & - & - & $\begin{array}{c}0.005(0.002 \text { to } \\
0.01)\end{array}$ & $<0.001$ & $38(35)$ & - & - & 0.03 (0.02 to 0.07$)$ & $<0.001$ \\
\hline
\end{tabular}

Constrained interaction between years from first injection to long term cessation and opiate substitution treatment exposure:

\begin{tabular}{llllllllll}
\hline$\ll 1-1$ & - & - & - & - & - & - & - & - & \\
\hline $2-10$ & - & - & - & - & - & - & - & - & 1.0 \\
\hline $11-15$ & - & - & - & - & - & - & - & - & $1.2(1.1$ to 1.4$)$ \\
\hline $16-38$ & - & - & - & - & - & - & - & - & $1.3(1.2$ to 1.5$)$ \\
\hline
\end{tabular}

$\mathrm{IQR}=$ interquartile range.

*Adjusted for sex, age first injection, year first injection, exposure to opiate substitution treatment, prison history, overdose, HIV status, and interaction for hazard of long term cessation between duration of injecting and exposure to opiate substitution treatment.

treatment might also increase survival and reduce morbidity through improving social functioning, reducing criminal activity, and maintaining regular contact between individuals and primary care services. ${ }^{30}$
HIV infection was concentrated among cohort participants recruited in the early 1980 s, and deaths related to $\mathrm{HIV}$ peaked before the introduction of more effective treatments. ${ }^{13} \mathrm{HIV}$ infection reduced 


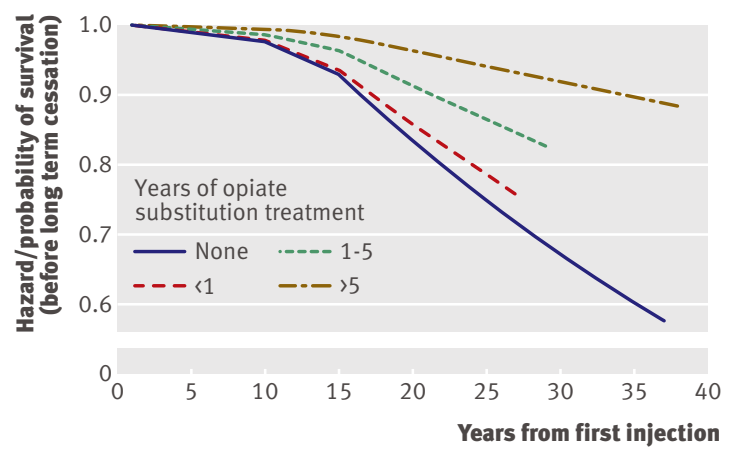

Fig 2 | Survival: probability of not dying before long term cessation by exposure to opiate substitution to treatment

survival overall and was associated with a sixfold increase in hazard of death before long term cessation. The risk of acquiring HIV infection associated with injecting in Scotland became widely known in $1986 .{ }^{12}$ After adjustment, our analyses suggest that participants who started injecting before 1986, and were perhaps more likely to have HIV positive contemporaries, had a shorter duration of injecting. ${ }^{3132}$

One long term cohort study found that episodes in prison seem to promote cessation of injection. ${ }^{33} \mathrm{We}$ found no evidence for this in our data; on the contrary, more than one prison episode almost doubled the overall hazard of death and decreased the probability of achieving long term cessation.

\section{Strengths and limitations}

The strengths of our study were its size, community base, and long duration of follow-up and the detailed information over time available from interview and administrative sources.

We lost 139 participants (18\% of the whole cohort and $25 \%$ of survivors), a favourable follow-up rate compared with many other cohorts of injecting drug users. ${ }^{34}$ Follow-up was poorer in those who had moved, in some cases because local general practitioners or directors of public health were unwilling to provide contact details. ${ }^{11}$ Those whom we failed to follow-up might have differed from the others and thus introduce bias. Unfortunately we were unable to conduct any imputation for missing data to test for bias because of a lack of information. Our participants might not be representative of all injecting drug users. Importantly, the cohort might over-represent dependent injecting drug users and under-represent those who inject for only a short time because recruitment occurred several years after onset and was based on users presenting to primary care and reporting their injecting drug use. In a sensitivity analysis, exclusion of participants who had injected for less than a year (who are less likely to be dependent or have a fatal overdose and more likely to stop injecting before dying than those who inject for over a year) had no effect on the hazard ratios reported in table 3. Overall survival and time to long term cessation in the population, however, might be underestimated and overestimated, respectively, if short term injectors were substantially under-represented. ${ }^{35}$ Equally, the cohort was at the epicentre of the national HIV epidemic, differing from other UK based cohorts of injecting drug users ${ }^{3637}$ but comparable with some European cohorts. ${ }^{132}$

The use of primary care records as our principal data source, on exposure and covariates and cessation outcomes in those who died, limited adjustment for potential confounders. Injecting drug users who do not enter opiate substitution treatment might have different characteristics (for example, be less chaotic or dependent) that explain their higher cessation rate and confound the relation between long term cessation and opiate substitution treatment. If that is true, we would also expect to see a lower risk of death among those who did not receive opiate substitution treatment, which is the reverse of what we observed. It is not inevitable that any factor, such as exposure to opiate substitution treatment, that reduces risk of death in injecting drug users will also increase overall duration of injecting. For example, prison history increased both mortality and injecting duration.

Information on injection cessation from interviews or patients' notes, or both, might have been biased. For example recipients of opiate substitution treatment might be more likely to have their injection status discussed and recorded in their notes than those receiving no active intervention for a previously declared drug problem, which might introduce differential bias and explain the apparent effects of opiate substitution treatment on long term cessation. We found the same relation between opiate substitution treatment exposure and long term cessation shown in figure 2, however, when we restricted the analysis to survivors with interview data.

Continued injecting among those who received opiate substitution treatment might reflect poor prescribing or inadequate doses of the substitute drug involved, both of which were associated with a higher risk of mortality in other studies in Scotland..$^{38}$ This seems unlikely. Doses prescribed at Muirhouse are typically high (current mean daily dose of methadone is $90 \mathrm{mg}$ ). Some opiate substitution treatment, particularly before the $1990 \mathrm{~s}$, could have been at lower doses. ${ }^{30}$

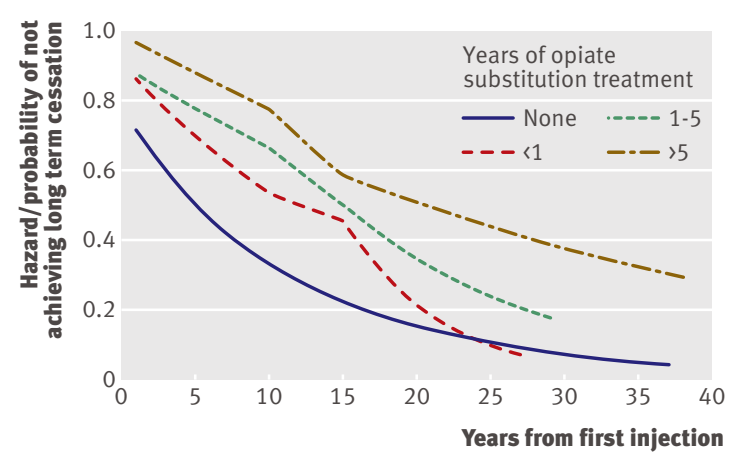

Fig 3 | Injecting duration: probability of achieving long term cessation by exposure to opiate substitution treatment 


\section{WHAT IS ALREADY KNOWN ON THIS TOPIC}

Injecting drug use is a chronic condition associated with substantial excess mortality and morbidity, but there is a lack of empirical evidence on the duration of injecting

Opiate substitution treatment reduces the risk of death in injectors over the short term, possibly by providing a faster route to full recovery and abstinence from dependency

\section{WHAT THIS STUDY ADDS}

Opiate substitution treatment, especially long term, reduces the risk of death before cessation in injectors

Opiate substitution treatment does not reduce the overall duration of injecting

Debates on the direction of drug policy and benefits of drug treatment should consider that there is a balance between saving lives and achieving abstinence

Nevertheless, this would not explain why those with no exposure to opiate substitution treatment have a shorter duration of injecting; and the substantial survival benefits seen with opiate substitution treatment, particularly when prescribed for longer periods, also seems inconsistent if opiate substitution treatment was prescribed at an inadequate dose.

\section{Conclusions}

Injecting opiate use is typically a chronic health problem with substantial adverse health consequences. These consequences are ameliorated in part by oral opiate substitution treatment, the beneficial effects of which seem to be more substantial the longer it is prescribed. These benefits, however, do not seem to be mediated through reductions in the overall duration of injecting and might be associated with prolonged duration, albeit at reduced frequency during treatment. The implication is that prescribing guidelines that emphasise the key importance of complete cessation of injection or suggest that opiate substitution treatment should be withheld from injecting drug users with evidence of continued injecting are inappropriate and indeed likely to increase mortality. ${ }^{39}$

We gratefully acknowledge the provision of data from the Scottish Prison Service (Jim Carney), Lothian and Borders Police Force, the General Register Office for Scotland, and Information Services Division of the Scottish Government and the daily support and accommodation of the study by the partners and staff of the Muirhouse Medical Group. Contributors: JRR is principal investigator of the Edinburgh Addiction Study. MH, JM, and JRR conceived the present follow-up study and obtained funding. LC and JMcK undertook the fieldwork. DDA oversaw the analytic strategy with JK and MH. LC assembled the dataset, organised the contact tracing and external sources, and transposed case records into the dataset and managed the essential administration for the study. JK undertook data cleaning and descriptive analyses with LC, JK, MH, and DDA. JK wrote the first draft of the paper with all authors contributing to the final draft. JRR is study guarantor.

Funding: The present follow-up study of the Edinburgh Addiction Cohort was supported by the Chief Scientist Office for Scotland (CZH/4/318). At the time they undertook this work JM and $\mathrm{MH}$ were supported by career scientist fellowship awards from the National Institute of Health Research. JK is supported by an Australian National Health and Medical Research (NHMRC) postdoctoral training fellowship. The National Centre in HIV Epidemiology and Clinical Research is core-funded by the Australian Government Department of Health and Ageing. LC and JMcK were funded by the chief scientist office grant ( $\mathrm{CZH} / 4 / 318)$, with the latter being seconded to the study by the community drug problem service, (CDPS), Lothian. DDA is funded by Health Protection Agency and the Medical Research Council (grant No U.1052.00.007).
Competing interests: All authors have completed the Unified Competing Interest form at www.icmje.org/coi_disclosure.pdf (available on request from the corresponding author) and all authors want to declare (1) Financial support for the submitted work from Chief Scientist Office for Scotland (above). All authors also declare (2) No financial relationships with commercial entities that might have an interest in the submitted work; (3) No spouses, partners, or children with relationships with commercial entities that might have an interest in the submitted work; (4) No non-financial interests that may be relevant to the submitted work. Ethical approval: This study was approved by Lothian health ethics committee (LREC/2003/7/12) and informed consent was given by all participants

Data sharing: Data on the sensitivity analysis and further interview data are available from the corresponding author.

1 Bargagli AM, Hickman M, Davoli M, Perucci CA, Schifano P, Buster M, et al. Drug-related mortality and its impact on adult mortality in eight European countries. Eur J Public Health 2006;16:198-202.

2 Degenhardt L, Hall W, Warner-Smith M. Using cohort studies to estimate mortality among injecting drug users that is not attributable to AIDS. Sex Transm Infect 2006;82:56-63.

3 Ward J, Mattick RP, Hall W. Methadone maintenance treatment and other opioid replacement therapies. Harwood Academic Press, 1997.

4 Hubbard RL, Craddock SG, Anderson J. Overview of 5-year follow-up outcomes in the drug abuse treatment outcome studies (DATOS). J Subst Abuse Treat 2003;25:125-34.

5 Gossop M, Marsden J, Stewart D, Kidd T. The National Treatment Outcome Research Study (NTORS): 4-5 year follow-up results. Addiction 2003:98:291-303.

6 Darke S, Ross J, Teesson M, Ali R, Cooke R, Ritter A, et al. Factors associated with 12 months continuous heroin abstinence: findings from the Australian Treatment Outcome Study (ATOS). I Subst Abuse Treat 2005;28:255-63.

7 Gowing LR, Farrell M, Bornemann R, Sullivan LE, Ali RL. Methadone treatment of injecting opioid users for prevention of HIV infection. J Gen Intern Med 2006;21:193-95.

8 Davoli M, Bargagli AM, Perucci CA, Schifano P, Belleudi V, Hickman M, et al. Risk of fatal overdose during and after specialist drug treatment: the VEdeTTE study, a national multi-site prospective cohort study. Addiction 2007;102:1954-9.

9 Termorshuizen F, Krol A, Prins M, Geskus R, van den Brink W, Van Ameijden EJ. Prediction of relapse to frequent heroin use and the role of methadone prescription: an analysis of the Amsterdam Cohort Study among drug users. Drug Alcohol Depend 2005;79:231-40.

10 McKeganey N. The lure and the loss of harm reduction in UK drug policy and practice. Addict Res Theory 2006;14:557-88.

11 Macleod J, Copeland L, Hickman M, McKenzie J, Kimber J, DeAngelis D, et al. The Edinburgh addiction cohort: recruitment and follow-up of a primary care based sample of injection drug users and non drug-injecting controls. BMC Public Health 2010;10:101.

12 Robertson JR, Bucknall AB, Welsby PD, Roberts JJ, Inglis JM, Peutherer JF, et al. Epidemic of AIDS related virus (HTLV-III/LAV) infection among intravenous drug abusers. BMJ 1986;292:527-9.

13 Copeland L, Budd J, Elton RA, Robertson JR. The changing patterns in causes of death in a cohort of injecting drug users 1980-2001. Arch Intern Med 2004;164:1214-20.

14 Blane D. Collecting retrospective data: development of a reliable method and a pilot study of its use. Soc Sci Med 1996;42:751-7.

15 Babor T, Higgins-Biddle JC, Saunders JS, Monteiro M. The alcohol us disorders identification test: guidelines for use in primary care. 2nd ed. WHO, 2001.

16 Zigmond AS, Snaith RP. The hospital anxiety and depression scale. Acta Psychiatr Scand 1983;67:361-70.

17 Rabin R, de Charro F. EQ-5D: a measure of health status from the EuroQol Group. Ann Med 2001;33:337-43.

18 Shah NG, Galai N, Celentano DD, Vlahov D, Strathdee SA. Longitudinal predictors of injection cessation and subsequent relapse among a cohort of injection drug users in Baltimore, MD, 1988-2000. Drug Alcohol Depend 2006;83:147-56

19 Langendam MW, Van Brussel G, Coutinho RA, Van Ameijden EJ. Methadone maintenance and cessation of injecting drug use: results from the Amsterdam Cohort Study. Addiction 2000;94:591-600.

20 Hser Y, Hoffman V, Grella C, Anglin D. A 33-year follow-up of narcotics addicts. Arch Gen Psychiatry 2001;58:503-8.

21 Robertson JR, Raab GM, Bruce M, McKenzie JS, Storkey HR, Salter A Addressing the efficacy of dihydrocodeine versus methadone as an alternative maintenance treatment for opiate dependence: a randomized controlled trial. Addiction 2006;101:1752-9.

22 Hosmer D, Lemeshow S. Applied survival analysis: regression modeling of time to event data. John Wiley, 1999.

23 Collet D. Modelling survival data in medical research. 2 nd ed. Chapman and Hall, 2003. 
24 Putter H, Fiocco M, Geskus RB. Tutorial in biostatistics: competing risks and multi-state models. Stat Med 2007;26:2389-430.

25 Allison PD. Discrete-time methods for the analysis of event histories. Soc Methodol 1982;13:61-98.

26 Berrington A, Diamond IM. Marriage or cohabitation: a competing risk analysis of first partnership formation among the 1958 British birth cohort. J R Stat Soc Ser A 2000;163:127-51.

27 O'Brien CP, McLellan AT. Myths about the treatment of addiction. Lancet 1996;347:237-40.

28 Advisory Council on the Misuse of Drugs. Treatment and rehabilitation. HMSO, 1982.

29 Gowing L, Farrell M, Bornemann R, Ali R. Substitution treatment of injecting opioid users for prevention of HIV infection. Cochrane Database Syst Rev 2008;2:CD004145.

30 Department of Health (England) and the devolved administrations. Drug misuse and dependence: UK guidelines on clinical management. Department of Health (England), the Scottish Government, Welsh Assembly Government, Northern Ireland Executive, 2007.

31 Skidmore CA, Robertson JR, Robertson AA, Elton RA. After the epidemic: follow up study of HIV seroprevalence and changing patterns of drug use. BMJ 1990;300:219-23.

32 Termorshuizen F, Krol A, Prins M, van Ameijden EJ. Long term outcome of chronic drug use-the Amsterdam cohort study among drug users. Am J Epidemiol 2005;161:271-9.
33 Vaillant GE. What can long-term follow-up teach us about relapse and prevention of relapse in addiction? BrJAddict 1988;83:1147-57.

34 Hartsock P, Genser S, eds. Longitudinal studies of HIV infection in intravenous drug users: methodological issues in natural history research. National Institute on Drug Abuse, 1991.

35 Sweeting MJ, De Angelis D, Ades AE, Hickman M. Estimating the prevalence of ex-injecting drug use in the population. Stat Methods Med Res 2009;18:381-95.

36 Rathod NH, Addenbrooke WM, Rosenbach AF. Heroin dependence in an English town: 33-year follow-up. Br J Psychiatry 2005;187:421-5.

37 Oppenheimer E, Tobutt C, Taylor C, Andrew T. Death and survival in a cohort of heroin addicts from London clinics: a 22-year follow-up study. Addiction 1994;89:1299-308.

38 McCowan C, Kidd B, Fahey T. Factors associated with mortality in Scottish patients receiving methadone in primary care: retrospective cohort study. BM/ 2009;338:b2225.

39 Fugelstad A, Stenbacka M, Leifman A, Nylander M, Thiblin I. Methadone maintenance treatment: the balance between life-saving treatment and fatal poisonings. Addiction 2007;102:406-12.

40 Kind P, Hardman G, Macran S. UK population norms for EQ-5D. York Centre for Health Economics, 1999 (discussion paper 172).

Accepted: 13 April 2010 